Marugán, Montserrat; Carbonero, Miguel Ángel; León, Benito; Galán, Manuel (2013). Análisis del uso de estrategiās de recuperación de la información por alumnos con alta capacidad intelectual (9-14 años) en función del género, edad, nivel educativo y creatividad. Revista de Investigación Educativa, 31 (1), 185-198.

http://dx.doi.org/10.6018/rie.31.1.147361

\title{
ANÁLISIS DEL USO DE ESTRATEGIAS DE RECUPERACIÓN DE LA INFORMACIÓN POR ALUMNOS CON ALTA CAPACIDAD INTELECTUAL (9-I 4 AÑOS) EN FUNCIÓN DEL GÉNERO, EDAD, NIVEL EDUCATIVOY CREATIVIDAD
}

\author{
Montserrat Marugán', Miguel Ángel Carbonero', Benito León² y Manuel Galán' \\ 'Universidad de Valladolid. \\ ¿Universidad de Extremadura
}

(España)

\section{RESUMEN}

En este trabajo se estudian las relaciones entre alta dotación intelectual y estrategias de recuperación de información, atendiendo a las influencias del género, la edad, el nivel educativo y la creatividad. Se evalúan las estrategias con las Escalas ACRA (Román y Gallego, 1994); la inteligencia general con el Test de Factor " $g$ " (Cattell y Cattell, 1990) y el Test de Matrices Progresivas (Raven, 1996) y la creatividad con el Test de Abreacción (TAEC) (De la Torre, 1991). Se utiliza una muestra de alumnos de alta dotación intelectual de Educación Primaria y Secundaria (9-14 años).

Los resultados no muestran relaciones significativas entre las variables inteligencia general y estrategias de recuperación de la información. La edad modula de forma limitada su uso y la asociación entre alta dotación y alta creatividad solo favorece la utilización de las estrategias

\footnotetext{
Correspondencia:

Montserrat Marugán de Miguelsanz. Departamento de Psicología. Facultad de Educación y Trabajo Social. Universidad de Valladolid. Paseo de Belén no 1. 47011, Valladolid. mmarugan@psi.uva.es

Miguel Ángel Carbonero Martín. Departamento de Psicología. Facultad de Educación y Trabajo Social. Universidad de Valladolid. Paseo de Belén oํ 1. 47011, Valladolid

Benito León del Barco. Departamento de Psicología y Antropología. Universidad de Extremadura. bleon@unex.es

Manuel Galán de la Calle. Departamento de Psicología. Facultad de Educación y Trabajo Social. Universidad de Valladolid. Paseo de Belén oํ 1. 47011, Valladolid. manu_galan79@hotmail.com

arboner@psi.uva.es
} 
de Respuesta Escrita. Se propone una revisión de las evaluaciones de uso percibido, frente a las de uso real.

Palabras clave: Estrategias de recuperación; Alta dotación; Estrategias de aprendizaje; Creatividad.

\title{
ANALYSIS OF THE USE OF INFORMATION RETRIEVAL STRATEGIES BY STUDENTS WITH HIGH INTELLECTUAL CAPABILITIES ACCORDING TO SEX,AGE, EDUCATIONAL LEVEL,AND CREATIVITY
}

\begin{abstract}
In this paper the relation between high intellectual capabilities and information retrieval strategies are studied. The variables are: sex, age, educational level and creativity. Strategies were measured with the ACRA Scales (Román and Gallego, 1994, 2004). General intelligence was measured with the " $g$ " Factor Test (Cattell and Cattell, 1990) and the Progressive Matrices Test (Raven, 1996). Creativity was analyzed using the TAEC Abreaction Test (de la Torre, 1991). Primary School and Compulsory Secondary Education students (aged 9-14) from Castilla y León (Spain) were chosen because of their high intellectual capability. Results show a significant association between general intelligence and information retrieval strategies. Age determines to some extent the use of strategies. The relation between a high intellectual capability and creativity only promotes the use of Written Answer strategies. A revision of evaluations of perceived use versus evaluations of real use of strategies is proposed.

Keywords: Retrieval strategies; High intellectual capability; Learning strategies; Creativity.
\end{abstract}

\section{INTRODUCCIÓN}

Los estudios sobre estrategias de aprendizaje puede considerarse una de las líneas de investigación más fructíferas desarrollada a lo largo de los últimos años dentro del ámbito del aprendizaje escolar y de los factores que inciden en el mismo. Han sido objeto de numerosas investigaciones y trabajos. García, Clemente y Pérez (2) llevaron a cabo un estudio sobre la evolución y desarrollo de las estrategias de aprendizaje en Psicología de la Educación utilizando como fuente bibliográfica el Psychological Literature de la A.P.A. (American Psychological Association). Encontraron un total de 272 artículos, publicados desde 1984 hasta 1991, en los que aparece el término Estrategias de aprendizaje (learning strategies) en su abstract, observando una clara expansión del estudio de este tema - de cinco artículos en 1984 se pasa a treinta y tres en 1986 y a setenta en 1990 .

En estos años y en las décadas posteriores se han realizado avances importantes en el diseño de modelos teóricos (Román y Gallego, 2004; Beltrán, 1996; Bernad, 1995; Monereo, 1993, Nisbet y Shucksmith, 1991); así como en la experimentación de procedimientos y programas de intervención (López, 1998; Marugán, 1996, 2009; Marugán y Román, 1997; Martín, Román y Carbonero, 2005).

Las estrategias de aprendizaje de los alumnos con superdotación intelectual, talentosos y/o de altas capacidades han sido investigadas en diferentes ocasiones (Gallego 
y Gonzalez, 2008), pero los resultados no son siempre coincidentes. Así, Román y Gallego (1994) afirma que los alumnos superdotados destacan en procesos y estrategias específicas de adquisición, codificación, recuperación y apoyo al procesamiento de la información de información.

Beltrán (1999) señala que los alumnos superdotados poseen mejores estrategias en tres principales aspectos (a) metacognitivas (planificación, seguimiento, evaluación), (b) cognitivas (selección, organización, elaboración de la información) y (c) mejores estrategias de apoyo (motivación, deseo de novedad, percepción de autoeficacia).

Prieto y Castejón (1999) sugieren que, en general, los sujetos de habilidad alta poseen un repertorio de estrategias más profundas y elaboradas que las de los sujetos de habilidades medias. Aunque no encuentran diferencias respecto a los de habilidades medias en muchas estrategias de planificación del estudio, selección de la información relevante mediante subrayado o toma de notas, ni en la organización de contenidos mediante la, esquemas o mapas conceptuales, realización de resúmenes, etc.

Domènech (2004) estudia con detenimiento el papel de la inteligencia y la metacognición en la resolución de problemas. Encuentra que no existen diferencias entre estudiantes de capacidad alta y estudiantes de capacidad media en ninguno de los tres aspectos de metacognición que estudia: experiencia cognitiva, conocimiento y eficacia metacognitiva.

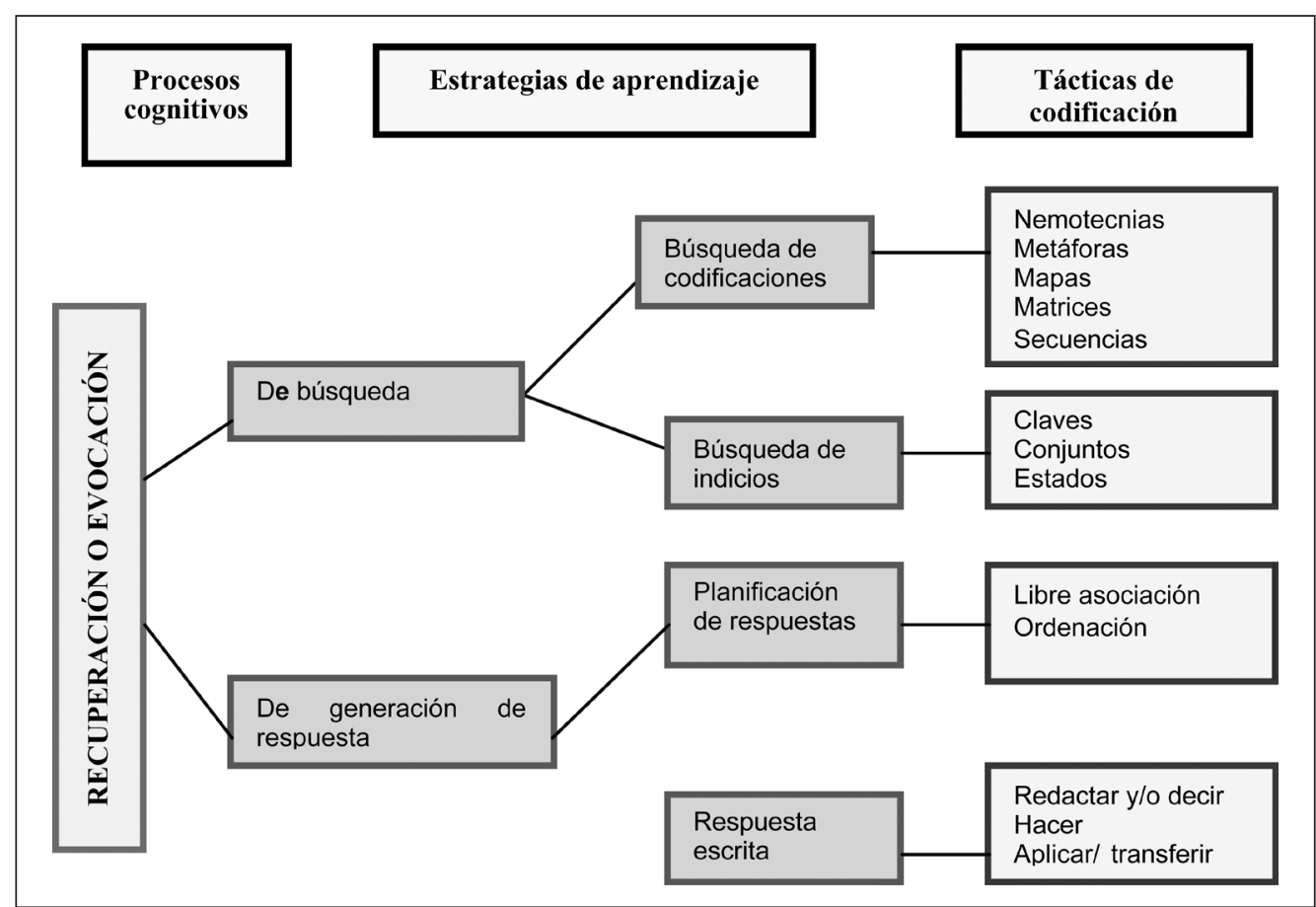

GRÁFICO 1

CLASIFICACIÓN DE LAS ESTRATEGIAS DE RECUPERACIÓN. ACRA. ESCALAS DE ESTRATEGIAS DE APRENDIZAJE (ROMÁN Y GALLEGO, 1994, 2004) 
En una investigación anterior (Del Caño, Marugán, Román, Galán y Torres, 2005), respecto a las diferencias entre estudiantes de alto CI y el resto de alumnos del grupo control (inteligencia media y baja) en lo que se refiere a estrategias generales de aprendizaje evaluadas mediante las escalas ACRA se comprobó que, puntuando mejor el grupo de CI alto en prácticamente todas las subescalas y en el total de la prueba, las diferencias no alcanzaban significación estadística. García, Prieto y Bermejo (2000) aplicando el mismo instrumento de evaluación (escalas ACRA) con alumnos de Bachillerato de alta dotación comprueban resultados en el mismo sentido $(p \leq 0,04)$.

Las estrategias de recuperación serían las encargadas de recordar y usar la información que ha sido previamente procesada. Favorecen la búsqueda de información en la memoria y la generación de respuesta. Pueden ser, por lo tanto, de dos tipos: a) estrategias de búsqueda, que trasforman la información desde la memoria a largo plazo a la memoria a corto plazo para generar respuestas. Van a depender del modo en que se codificó la información, y b) estrategias de generación de respuesta, que se encargarían de planificar el modo en que se va a utilizar la información, (ver gráfico 1).

\section{OBJETIVOS}

Los objetivos que se pretenden conseguir con este estudio son los siguientes: (a) Evaluar, en una muestra de alumnos de Primaria y Secundaria, mediante medidas de autoinforme, las estrategias de recuperación de información que dicen utilizar los alumnos de altas capacidades; (b) analizar las relaciones que se establecen entre las variables uso de estrategias de recuperación y puntuaciones obtenidas en inteligencia general (CI) y (c) examinar factores moduladores (género, edad, nivel educativo y creatividad) que puedan influir en la utilización de las estrategias de recuperación de información en alumnos de alta dotación.

\section{METODOLOGÍA}

\section{Participantes}

En una muestra de alumnos de Castilla y León, seleccionada al azar para su participación en un proyecto de identificación de alumnos superdotados, se toma para este trabajo una representación de 327 alumnos de cuatro centros educativos. Estos centros poseen un porcentaje alto de alumnos talentosos. Concretamente entre los cuatro se identifican 50 alumnos de altas capacidades, que es la muestra que se considera para este estudio. Se nominan sujetos de alta capacidad intelectual a los que puntúan con un CI superior o igual al percentil 95. Estos 50 alumnos de la muestra poseen un CI medio de 131,48 (desviación típica =6,459), oscilando entre 126 y 149 en las pruebas.

Son 21 varones y 29 mujeres, con edades comprendidas entre los 9 y 14 años, que se corresponden con las etapas educativas de $4^{\circ}, 5^{\circ}$ y $6^{\circ}$ de Educación Primaria y $1^{\circ}$ y $2^{\underline{o}}$ de Educación Secundaria Obligatoria (gráfico 2). Pertenecen a dos centros públicos y a dos concertados, con un nivel sociocultural medio. 


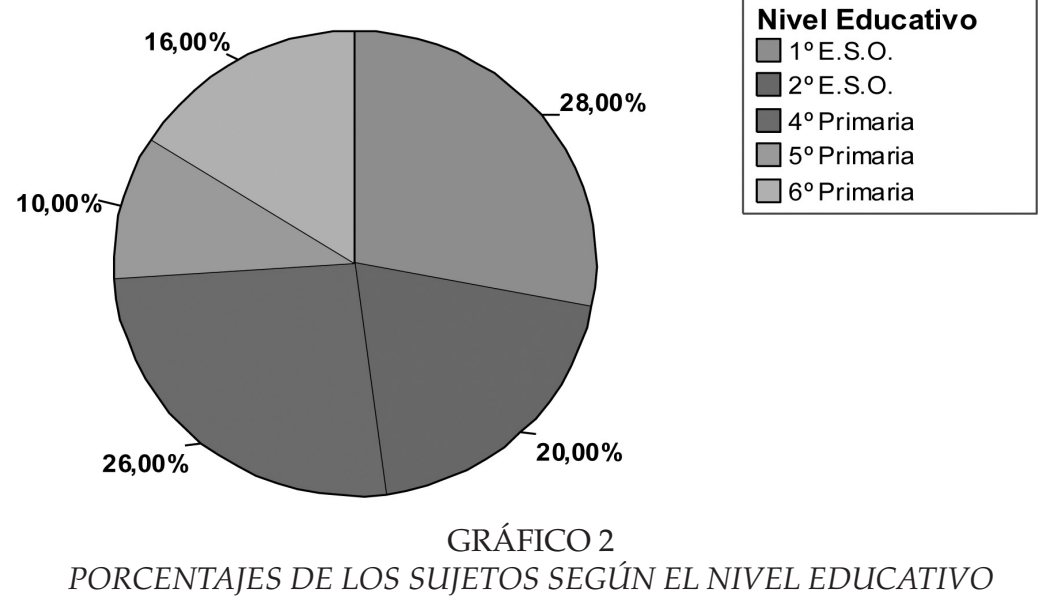

\section{Instrumentos}

Los datos se obtuvieron a través de cuatro pruebas estandarizadas y validadas:

Test de Factor "g" de Inteligencia. Escala 2. R.B. Cattell y A. K. S. Cattell (1990). Es una prueba que mide la inteligencia general no verbal. De aplicación colectiva, está tipificada para población española. Consta de tres escalas para distintas edades, en esta investigación se aplicó la Escala 2 (forma B). Cada escala consta de cuatro pruebas: Series, Clasificaciones, Matrices y Condiciones.

Test de Matrices Progresivas. J. C. Raven (1996). Se trata de una prueba no verbal destinada a medir la capacidad intelectual (habilidad mental general) para comparar formas y razonar por analogía, con independencia de los conocimientos adquiridos y de la cultura del individuo. Los ítems que conforman este test se denominan matrices porque se asemejan a determinantes matemáticos. Y reciben el apelativo d progresivas porque la secuencia de dibujos desarrolla hábitos de atención, percepción y pensamiento que permiten a los sujetos resolver, con mayor facilidad, elementos más difíciles. Escalas ACRA de Estrategias de Aprendizaje. Román y Gallego $(1994,2004)$ de evaluación del uso (percibido) de estrategias de aprendizaje. Versión modificada. Adaptación realizada por Marugán (1995) para alumnos en edad de cursar la escolaridad obligatoria.

ACRA consta de cuatro escalas y es el acrónimo de Adquisición, Codificación, Recuperación de información y Apoyo al procesamiento. Cada una de estas escalas es independiente, posee sus propios baremos, de tal forma que la prueba puede aplicarse en su totalidad o segmentada en escalas, dependiendo del diagnóstico que se quiera realizar. En este caso se han aplicado las cuatro escalas, aunque se analizan con más detalle en este trabajo los resultados referidos a la Escala de Recuperación. La Escala elegida evalúa, a su vez, las estrategias de recuperación de la información a través de cuatro subescalas (gráfico 1), las claves utilizadas son:

B.C.= Búsqueda de Codificaciones.

B.I = Búsqueda de Indicios.

P.R. = Planificación de Respuestas. 
R.E. = Respuesta Escrita.

E.R. = Total en Estrategias de Recuperación de Información.

En las respuestas de los alumnos a los ítem centrados en este tipo de estrategias se pretende, por ejemplo, obtener datos sobre la forma en que han codificado la información y si ésta es utilizada o favorece su recuperación; si les ayuda a recordar el evocar sucesos, episodios o anécdotas ocurridos en la clase o en los momentos de aprendizaje; si cuando les preguntan en un examen recuerdan palabras, dibujos o imágenes que tengan que ver con las ideas principales del material estudiado o si preparan la exposición o el examen previamente a nivel mental o con guiones, etc. En definitiva se trata de ver si hay una intencionalidad en la recuperación de la información y si se usan habitualmente estrategias para este fin.

Test de Abreacción para Evaluar la Creatividad (TAEC). (De la Torre, 1991). Se recomienda la utilización de la forma B desde la Educación Primaria en adelante, ya que las figuras a completar son algo más complejas (con mayor número de aperturas). Es una prueba de respuesta gráfica que permite valorar cuantitativamente la creatividad. Se puede aplicar desde los tres años de forma colectiva o individual. La tarea consiste en terminar 12 figuras inacabadas, variadas y poco convencionales. Proporciona una puntuación global y puntuaciones para nueve factores de creatividad. Los factores son: resistencia al cierre de aberturas, originalidad, elaboración de las figuras, fantasía, alcance imaginativo, expansión de las figuras, conectividad de unas figuras con otras, riqueza expresiva o vitalidad de las composiciones y fluidez gráfica. El test ofrece baremación en centiles por niveles educativos.

TABLA 1

RESUMEN DE LOS FACTORES DE CREATIVIDAD VALORADOS EN LA PRUEBA TAEC

\begin{tabular}{|c|c|c|}
\hline Factores de creatividad & Qué valora & Qué se puntúa \\
\hline Resistencia al cierre & $\begin{array}{l}\text { Capacidad para retrasar el cierre de } \\
\text { aperturas en la figura }\end{array}$ & $\begin{array}{l}\text { Aperturas abiertas o cerradas indi- } \\
\text { rectamente }\end{array}$ \\
\hline Originalidad & $\begin{array}{l}\text { Rareza estadística del contenido de } \\
\text { la imagen elaborada }\end{array}$ & $\begin{array}{l}\text { Infrecuencia de la imagen represen- } \\
\text { tada. }\end{array}$ \\
\hline Elaboración & $\begin{array}{l}\text { Enriquecimiento de la imagen con } \\
\text { detalles secundarios y adicionales }\end{array}$ & $\begin{array}{l}\text { Aparición de detalles adicionales, no } \\
\text { necesarios para interpretar la imagen }\end{array}$ \\
\hline Fantasía & $\begin{array}{l}\text { Contenido de la imagen inusual, } \\
\text { alejado del entorno familiar }\end{array}$ & $\begin{array}{l}\text { Grado en que la imagen se aleja del } \\
\text { entorno cotidiano }\end{array}$ \\
\hline Conectividad & $\begin{array}{l}\text { Integración de dos o más figuras en } \\
\text { un todo }\end{array}$ & Número de figuras conectadas \\
\hline Alcance imaginativo & $\begin{array}{l}\text { Lugar que ocupa en la imagen la } \\
\text { figura dada: cuerpo principal o ele- } \\
\text { mento secundario }\end{array}$ & $\begin{array}{l}\text { Grado en que la figura es un elemen- } \\
\text { to secundario de la imagen }\end{array}$ \\
\hline Expansión figurativa & $\begin{array}{l}\text { Espacio ocupado por la imagen ela- } \\
\text { borada }\end{array}$ & $\begin{array}{l}\text { Grado en que la imagen trasciende el } \\
\text { espacio de cada figura }\end{array}$ \\
\hline Riqueza expresiva & $\begin{array}{l}\text { Colorido, perspectiva y representa- } \\
\text { ción de seres vivos o en movimiento }\end{array}$ & $\begin{array}{l}\text { Presencia de color, volumen o de se- } \\
\text { res vivos o en movimiento }\end{array}$ \\
\hline $\begin{array}{l}\text { Coeficiente de fluidez } \\
\text { gráfica }\end{array}$ & $\begin{array}{l}\text { Capacidad de dar respuestas valio- } \\
\text { sas en el menor tiempo }\end{array}$ & $\begin{array}{l}\text { Cociente entre las puntuaciones de } \\
\text { los factores anteriores y el tiempo de } \\
\text { realización }\end{array}$ \\
\hline
\end{tabular}

Fuente: elaboración propia 


\section{Diseño y procedimiento}

Hemos seguido un diseño descriptivo correlacional. Se ha intentado controlar algunas variables extrañas que podrían producir algún efecto sobre los resultados finales: (i) Todas las pruebas utilizadas para la medición de CI, creatividad y estrategias de aprendizaje fueron aplicadas colectivamente en las mismas aulas donde los alumnos desarrollaban su vida diaria y durante el horario escolar normal. (ii) Se procuró que las diferentes pruebas fueran aplicadas en una parte del curso escolar en la que los alumnos no tuvieran ningún examen, para, de esta forma, controlar pérdidas de atención. (iii) El profesor, que en el momento de la prueba estaba dando clase, permanecía en el aula como sujeto tranquilizador al entrar un examinador extraño (un psicólogo del equipo).

\section{RESULTADOS}

En la tabla 2 mostramos un análisis descriptivo de las variables que analizaremos a lo largo de este estudio.

TABLA 2

ESTADÍSTICOS DESCRIPTIVOS

\begin{tabular}{|l|c|c|c|c|c|}
\cline { 2 - 5 } \multicolumn{1}{c|}{} & Media & $\begin{array}{c}\text { Desviación } \\
\text { Típica }\end{array}$ & Mínimo & Máximo & N \\
\hline CI & 131,48 & 6,459 & 126 & 149 & 50 \\
\hline Creatividad & 86,26 & 33,971 & 9 & 159 & 50 \\
\hline Estrategias de adquisición & 23,64 & 3,403 & 15 & 32 & 50 \\
\hline Estrategias de codificación & 58,74 & 8,921 & 35 & 78 & 50 \\
\hline Estrategias metacognitivas & 14,88 & 3,101 & 7 & 20 & 50 \\
\hline Estrategias de apoyo o socioafectivas & 29,18 & 4,119 & 15 & 36 & 50 \\
\hline Estrategias de recuperación & 23,24 & 2,945 & 16 & 28 & 50 \\
\hline (B.C.) Búsqueda de codificaciones & 3,48 &, 677 & 2 & 4 & 50 \\
\hline (B.I.) Búsqueda de indicios & 3,14 &, 756 & 1 & 4 & 50 \\
\hline (P.R.) Planificación de respuesta & 3,66 &, 626 & 2 & 4 & 50 \\
\hline (R.E.) Respuesta escrita & 12,92 & 2,088 & 8 & 16 & 50 \\
\hline Estrategias de aprendizaje & 149,68 & 18,423 & 119 & 194 & 50 \\
\hline
\end{tabular}

Ante la pregunta de si existe relación funcional asociativa entre las puntuaciones en inteligencia general y las obtenidas en estrategias de recuperación de información en la muestra de alumnos de alta dotación, según nuestros resultados (tabla 3), la variable inteligencia general no correlaciona significativamente con ninguna de las estrategias de recuperación de información. Hay que hacer constar que en los análisis realizados entre la variable CI y el resto de estrategias de aprendizaje de la Escala ACRA tampoco se encuentran correlaciones significativas, así con respecto a las estrategias de adquisición la correlación es de -,151; a las de codificación $(-, 028)$; estrategias metacognitivas $(, 177)$ y respecto a las estrategias de apoyo $(-, 046)$. 
TABLA 3

CORRELACIÓN (R DE PEARSON) ENTRE CI Y ESTRATEGIAS DE RECUPERACIÓN DE INFORMACIÓN

\begin{tabular}{|c|c|c|c|c|c|c|c|}
\cline { 2 - 7 } \multicolumn{2}{c|}{} & CI & $\begin{array}{c}\text { Búsqueda } \\
\text { codificación }\end{array}$ & $\begin{array}{c}\text { Búsqueda } \\
\text { indicios }\end{array}$ & $\begin{array}{c}\text { Planificación } \\
\text { respuesta }\end{array}$ & $\begin{array}{c}\text { Respuesta } \\
\text { escrita }\end{array}$ & $\begin{array}{c}\text { Total E. } \\
\text { Recuperac. }\end{array}$ \\
\hline \multirow{3}{*}{$\mathrm{CI}$} & $\begin{array}{c}\text { Correlación } \\
\text { de Pearson }\end{array}$ & 1 &, 021 &, 141 &, 051 &,- 074 &,- 004 \\
\cline { 2 - 8 } & Sig. (bilateral) & $\cdot$ &, 885 &, 330 &, 724 &, 608 &, 978 \\
\cline { 2 - 8 } & $\mathrm{N}$ & 50 & 50 & 50 & 50 & 50 & 50 \\
\hline
\end{tabular}

Hemos categorizado las puntuaciones en inteligencia general de los sujetos con altas capacidades en 3 grupos para ver si existe relación funcional causal entre aquella y las estrategias de recuperación de información (media en la tabla 4), pero como podemos observar en dicha tabla, tampoco aquí hemos encontrado un valor significativo que justifique dicha atribución.

De forma colateral observamos que existe una correlación significativa $(p \leq 0,01)$ entre la puntuación total en Estrategias de Recuperación de Información y las distintas subestrategias de recuperación de información.

TABLA 4

ANOVA TOMANDO EL CI COMO VARIABLE INDEPENDIENTE

( $1=$ alto, $2=$ medio, $3=$ bajo $)$

\begin{tabular}{|c|c|c|c|c|c|c|c|c|c|}
\hline & & $\mathbf{N}$ & Media & Desviación típica & Mínimo & Máximo & gl & $\mathbf{F}$ & Sig. \\
\hline \multirow{3}{*}{ B.C } & 1 & 17 & 3,41 & ,795 & 2 & 4 & \multirow{3}{*}{2} & \multirow{3}{*}{,329 } & \multirow{3}{*}{,721 } \\
\hline & 2 & 10 & 3,40 & ,516 & 3 & 4 & & & \\
\hline & 3 & 23 & 3,57 & ,662 & 2 & 4 & & & \\
\hline \multirow{3}{*}{ B.I. } & 1 & 17 & 3,24 & ,562 & 2 & 4 & \multirow{3}{*}{2} & \multirow{3}{*}{,299 } & \multirow{3}{*}{,743 } \\
\hline & 2 & 10 & 3,00 & 816 & 2 & 4 & & & \\
\hline & 3 & 23 & 3,13 & 869 & 1 & 4 & & & \\
\hline \multirow{3}{*}{ P.R. } & 1 & 17 & 3,71 & ,588 & 2 & 4 & \multirow{3}{*}{2} & \multirow{3}{*}{,399 } & \multirow{3}{*}{,673 } \\
\hline & 2 & 10 & 3,50 & 707 & 2 & 4 & & & \\
\hline & 3 & 23 & 3,70 & 635 & 2 & 4 & & & \\
\hline \multirow{3}{*}{ R.E. } & 1 & 17 & 12,65 & 1,656 & 9 & 15 & \multirow{3}{*}{2} & \multirow{3}{*}{,729 } & \multirow{3}{*}{,488 } \\
\hline & 2 & 10 & 12,50 & 2,121 & 9 & 16 & & & \\
\hline & 3 & 23 & 13,30 & 2,363 & 8 & 16 & & & \\
\hline \multirow{3}{*}{ E.R. } & 1 & 17 & 23,00 & 2,739 & 17 & 27 & \multirow{3}{*}{2} & \multirow{3}{*}{,557 } & \multirow{3}{*}{,576 } \\
\hline & 2 & 10 & 22,60 & 2,591 & 18 & 27 & & & \\
\hline & 3 & 23 & 23,70 & 3,267 & 16 & 28 & & & \\
\hline
\end{tabular}

Cabría preguntarse cómo se comportan la variable CI y las estrategias de aprendizaje en general y de recuperación en particular, cuando consideramos a los estudiantes de alta y no alta capacidad intelectual. En este sentido se ha comprobado (tabla 5) que, puntuando mejor el grupo de CI alto en prácticamente todas las subescalas de estrategias y en el total de la prueba ACRA, las diferencias no alcanzan significación estadística a excepción de los resultados en la Escala de estrategias de recuperación 
de la información. Es decir, los alumnos de alta capacidad intelectual sí superan en uso de estrategias de recuperación a los alumnos menos dotados, aunque cuando se considera únicamente la muestra de estudiantes de alta dotación no son relevantes las diferencias encontradas.

TABLA 5

DESCRIPTIVOS CORRESPONDIENTES A LOS RESULTADOS EN LA PRUEBA ACRA Y DIFERENCIAS ENTRE LOS GRUPOS DE CI ALTO (1) Y NO ALTO (2)

\begin{tabular}{|l|c|c|c|c|c|}
\cline { 2 - 6 } \multicolumn{1}{c|}{} & CI & N & Media & $\begin{array}{c}\text { Desviación } \\
\text { típica }\end{array}$ & Sig. \\
\hline \multirow{2}{*}{ Estrategias de adquisición } & 1 & 54 & 23,35 & 3,43 & \\
\cline { 2 - 6 } & 2 & 251 & 22,61 & 3,65 & N.S. \\
\hline \multirow{2}{*}{ Estrategias de codificación } & 1 & 54 & 58,37 & 8,96 & \\
\cline { 2 - 6 } & 2 & 251 & 57,33 & 8,13 & N.S. \\
\hline \multirow{2}{*}{ Estrategias de recuperación } & 1 & 54 & 23,13 & 3,05 & \\
\cline { 2 - 6 } & 2 & 251 & 21,98 & 3,11 & 0.014 \\
\hline \multirow{2}{*}{ Estrategias metacognitivas } & 1 & 54 & 15,00 & 3,03 & \\
\cline { 2 - 6 } & 2 & 251 & 15,28 & 2,60 & N.S. \\
\hline \multirow{2}{*}{ Estrategias de apoyo } & 1 & 54 & 29,67 & 3,50 & \\
\cline { 2 - 6 } & 2 & 251 & 28,61 & 4,15 & .084 \\
\hline \multirow{2}{*}{ TOTAL } & 1 & 54 & 149,52 & 18,16 & \\
\cline { 2 - 6 } & 2 & 251 & 145,82 & 17,75 & N.S. \\
\hline Estrategias de adquisición & & 327 & 22,79 & 3,60 & \\
Estrategias de codificación & & 327 & 57,73 & 8,38 & \\
Estrategias de recuperación & Total & 327 & 22,31 & 3,15 & \\
Estrategias metacognitivas & Muestra & 327 & 15,32 & 2,69 & \\
Estrategias de apoyo & & 327 & 28,95 & 4,02 & \\
TOTAL & & 327 & 147,10 & 17,96 & \\
\hline
\end{tabular}

Para comprobar si existe relación funcional causal entre el nivel educativo (edad) y las estrategias de recuperación de información hemos categorizado el nivel educativo de los sujetos con altas capacidades en 4 grupos. En este caso, como se aprecia en la tabla 6, tampoco hemos encontrado ninguna relación significativa. Los alumnos de altas capacidades puntúan en esta estrategia de forma similar estando en uno u otro nivel educativo. La edad no parece ser discriminante, aunque con la subestrategia de Respuesta Escrita (R.E.) sí parece existir una tendencia a la relación causal $(p=0,099)$, ya que los alumnos del primer nivel ( 9 años) puntúan ligeramente inferior.

Además se obtuvieron los siguientes resultados cuando se consideraron las puntuaciones en el total de la escala ACRA: (a) Relación funcional causal significativa $(p \leq 0,05)$ entre el nivel educativo y estos dos tipos de estrategias: las estrategias de codificación $(p=0,54)$ y las estrategias de apoyo al procesamiento $(p=0,01)$. (b) Tendencia a la relación funcional causal $(p=0,064)$ entre el nivel educativo y la puntuación total en Estrategias de Aprendizaje, confirmando estudios anteriores (Del Caño, Marugán, Román, Galán y Torres, 2005). 
TABLA 6

ANOVA TOMANDO EL NIVEL EDUCATIVO (EDAD) COMO VARIABLE INDEPENDIENTE

$\left(1=4^{\circ}\right.$ Ed. Primaria, $2=5^{\circ}$ y $6^{\circ}$ Ed. Primaria, $3=1^{\circ}$ E.S.O, $4=2^{\circ}$ E.S.O $)$

\begin{tabular}{|c|c|c|c|c|c|c|c|c|c|}
\hline & & $\mathbf{N}$ & Media & $\begin{array}{l}\text { Desv. } \\
\text { típica }\end{array}$ & Mínimo & Máximo & gl & $\mathbf{F}$ & Sig. \\
\hline \multirow{4}{*}{ B.C } & 1 & 13 & 3,62 & ,506 & 3 & 4 & \multirow{4}{*}{3} & \multirow{4}{*}{2,039} & \multirow{4}{*}{,122 } \\
\hline & 2 & 13 & 3,77 & ,599 & 2 & 4 & & & \\
\hline & 3 & 14 & 3,21 & 802 & 2 & 4 & & & \\
\hline & 4 & 10 & 3,30 & ,675 & 2 & 4 & & & \\
\hline \multirow{4}{*}{ B.I. } & 1 & 13 & 3,23 & ,927 & 1 & 4 & \multirow{4}{*}{3} & \multirow{4}{*}{,265 } & \multirow{4}{*}{850} \\
\hline & 2 & 13 & 3,23 & ,725 & 2 & 4 & & & \\
\hline & 3 & 14 & 3,07 &, 475 & 2 & 4 & & & \\
\hline & 4 & 10 & 3,00 & ,943 & 2 & 4 & & & \\
\hline \multirow{4}{*}{ P.R. } & 1 & 13 & 3,77 & 439 & 3 & 4 & \multirow{4}{*}{3} & \multirow{4}{*}{,559 } & \multirow{4}{*}{ 645 } \\
\hline & 2 & 13 & 3,77 & ,599 & 2 & 4 & & & \\
\hline & 3 & 14 & 3,57 & 646 & 2 & 4 & & & \\
\hline & 4 & 10 & 3,50 & ,850 & 2 & 4 & & & \\
\hline \multirow{4}{*}{ R.E. } & 1 & 13 & 11,85 & 2,304 & 8 & 16 & \multirow{4}{*}{3} & \multirow{4}{*}{2,216} & \multirow{4}{*}{ 099 } \\
\hline & 2 & 13 & 13,85 & 2,375 & 8 & 16 & & & \\
\hline & 3 & 14 & 12,86 & 1,167 & 11 & 15 & & & \\
\hline & 4 & 10 & 13,20 & 2,044 & 10 & 16 & & & \\
\hline \multirow{4}{*}{ E.R. } & 1 & 13 & 22,62 & 3,070 & 16 & 28 & \multirow{4}{*}{3} & \multirow{4}{*}{1,338} & \multirow{4}{*}{,274 } \\
\hline & 2 & 13 & 24,62 & 3,097 & 19 & 28 & & & \\
\hline & 3 & 14 & 22,71 & 1,858 & 19 & 26 & & & \\
\hline & 4 & 10 & 23,00 & 3,621 & 17 & 27 & & & \\
\hline
\end{tabular}

TABLA 7

ANOVA TOMANDO EL GÉNERO COMO VARIABLE INDEPENDIENTE

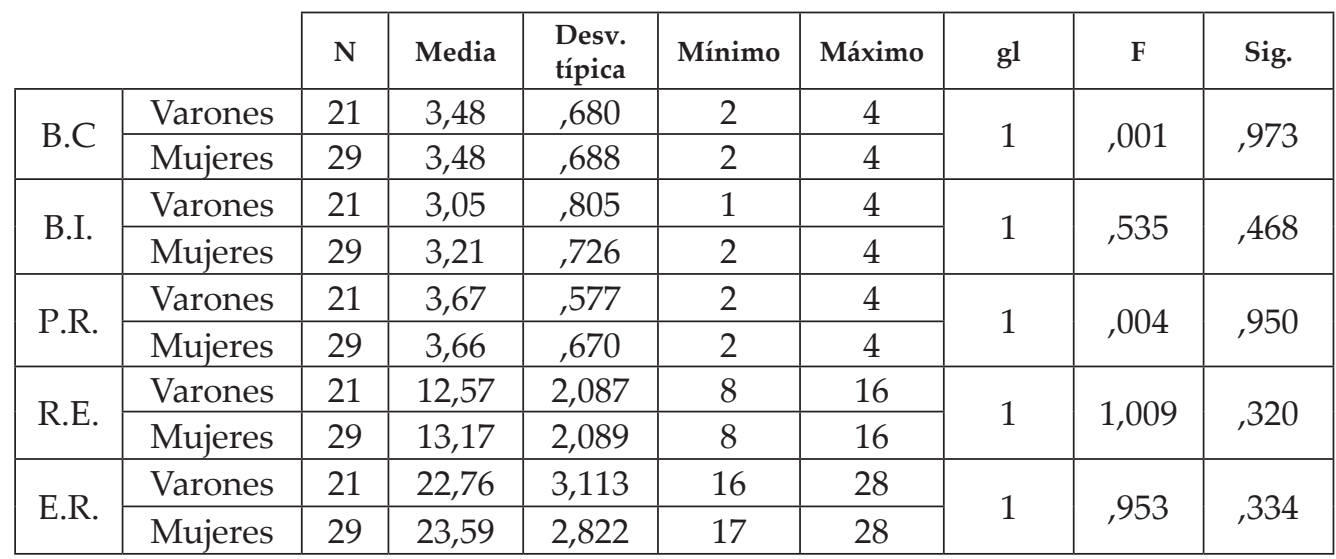


En la tabla 7 describimos la variable género respecto a las estrategias de recuperación de información. Según los resultados encontrados no existe ninguna relación funcional causal significativa entre la variable independiente género y las distintas subestrategias de recuperación de la información en alumnos de alta capacidad intelectual.

Del mismo modo se ha categorizado la puntuación total de creatividad en 3 grupos y tras un análisis ANOVA con esta variable como independiente se observan los siguientes resultados de interés (tabla 8). Entre la puntuación total en creatividad y la puntuación total en Estrategias de Recuperación de la Información (E.R.) existe una relación significativa ( $\mathrm{p} \leq 0,05)$; que se concreta únicamente entre la puntuación total en creatividad y la puntuación en estrategias de Respuesta Escrita (R.E.) ( $p=0,05)$.

TABLA 8

ANOVA TOMANDO COMO VARIABLE INDEPENDIENTE LA PUNTUACIÓN TOTAL EN CREATIVIDAD

(1= alta, $2=$ media, $3=$ baja $)$

\begin{tabular}{|c|c|c|c|c|c|c|c|c|c|}
\hline & & $\mathbf{N}$ & Media & Desv. típica & Mínimo & Máximo & gl & F & Sig. \\
\hline \multirow{4}{*}{ B.C } & 1 & 17 & 3,35 & ,702 & 2 & 4 & \multirow{4}{*}{2} & \multirow{4}{*}{1,941} & \multirow{4}{*}{ 155 } \\
\hline & 2 & 17 & 3,35 & ,702 & 2 & 4 & & & \\
\hline & 3 & 16 & 3,75 & ,577 & 2 & 4 & & & \\
\hline & Total & 50 & 3,48 & ,677 & 2 & 4 & & & \\
\hline \multirow{4}{*}{ B.I. } & 1 & 17 & 3,24 & 437 & 3 & 4 & \multirow{4}{*}{2} & \multirow{4}{*}{1,574} & \multirow{4}{*}{ 218 } \\
\hline & 2 & 17 & 2,88 & 857 & 2 & 4 & & & \\
\hline & 3 & 16 & 3,31 & 873 & 1 & 4 & & & \\
\hline & Total & 50 & 3,14 & ,756 & 1 & 4 & & & \\
\hline \multirow{4}{*}{ P.R. } & 1 & 17 & 3,59 & ,712 & 2 & 4 & \multirow{4}{*}{2} & \multirow{4}{*}{,689 } & \multirow{4}{*}{,507 } \\
\hline & 2 & 17 & 3,59 & ,712 & 2 & 4 & & & \\
\hline & 3 & 16 & 3,81 & ,403 & 3 & 4 & & & \\
\hline & Total & 50 & 3,66 & ,626 & 2 & 4 & & & \\
\hline \multirow{4}{*}{ R.E. } & 1 & 17 & 13,47 & 1,663 & 10 & 16 & \multirow{4}{*}{2} & \multirow{4}{*}{3,079} & \multirow{4}{*}{,055 } \\
\hline & 2 & 17 & 11,94 & 1,952 & 8 & 15 & & & \\
\hline & 3 & 16 & 13,38 & 2,363 & 8 & 16 & & & \\
\hline & Total & 50 & 12,92 & 2,088 & 8 & 16 & & & \\
\hline \multirow{4}{*}{ E.R. } & 1 & 17 & 23,65 & 2,644 & 17 & 27 & \multirow{4}{*}{2} & \multirow{4}{*}{3,894} & \multirow{4}{*}{,027 } \\
\hline & 2 & 17 & 21,76 & 2,587 & 18 & 27 & & & \\
\hline & 3 & 16 & 24,38 & 3,117 & 16 & 28 & & & \\
\hline & Total & 50 & 23,24 & 2,945 & 16 & 28 & & & \\
\hline
\end{tabular}

\section{DISCUSIÓNY CONCLUSIONES}

A la luz de los resultados obtenidos, no se produce ninguna relación funcional asociativa de carácter significativo entre las puntuaciones obtenidas por los alumnos de altas capacidades en inteligencia general y en estrategias de recuperación de información evaluadas con las Escalas ACRA de estrategias de aprendizaje. Hay que dejar 
constancia, sin embargo, que los alumnos de alta capacidad frente a los que no la poseen, sí puntúan de forma superior en todas las estrategias de aprendizaje, incluidas las estrategias de recuperación.

La variable género, según el estudio, no condiciona ningún efecto diferencial significativo en el uso de las cuatro estrategias de recuperación del modelo ACRA: Búsqueda de Codificaciones, Búsqueda de Indicios, Planificación de Respuestas y Respuesta Escrita.

Entre la variable nivel educativo (edad) y las puntuaciones en las estrategias de recuperación Respuesta Escrita existe una tendencia a la relación funcional causal ( $\mathrm{p}=$ 0,099), parece que cuanto mayor sea el nivel educativo y la edad de los sujetos, mejor será la planificación de la respuesta en el examen. El nivel educativo se ha mostrado también significativo $(\mathrm{p}=0,064)$ al compararle con la puntuación total en la Escala de estrategias generales de aprendizaje. La edad parece mejorar la eficacia en las estrategias ACRA.

Las relaciones entre creatividad e inteligencia son analizadas en numerosas investigaciones (López y Navarro, 2010; Preckel, Holling y Wiese, 2006); Sligh, Conners y Roskos, 2005). Por los resultados obtenidos la suma de estos dos factores no parece tener influencia en el uso percibido de estrategias de aprendizaje. Se muestra una relación significativa ( $\mathrm{p} \leq 0,05)$ entre creatividad y la puntuación total en Estrategias de Recuperación de la Información. Sin embargo no puede decirse que sea el grupo de alumnos intelectualmente bien dotado y con las mejores puntuaciones en creatividad el que obtenga las mejores puntuaciones en estrategias que permitan recuperar la información. Alta dotación intelectual más creatividad sólo ofrecería como resultado una mejor utilización de las estrategias de recuperación de Respuesta Escrita $(p \leq 0,05)$.

Aunque existe un consenso entre los estudiosos de estrategias de aprendizaje respecto al buen uso que de ellas hacen los alumnos con buena dotación intelectual, la realidad es que los resultados que se encuentran para avalar esta hipótesis, como también hemos podido ver en este estudio, no son concluyentes. Así, por ejemplo Del Caño, Marugán, Román, Galán y Torres (2005), estudiaron las relaciones entre la puntuación de la variable inteligencia general y el resto de estrategias de aprendizaje de la escala ACRA. No se encontraron interacciones interesantes, con la excepción de la referida a la subescala Estrategias de Apoyo al Procesamiento de la información ( $p \leq 0,05)$.

Como interpretación de los resultados de este estudio hay que tener en cuenta que las puntuaciones en inteligencia general de la muestra sufren pocas oscilaciones, ya que varían entre un CI de 126, puntuación mínima y 149 la puntuación máxima. No obstante en el trabajo anteriormente citado, con una muestra muy amplia de alumnos de alta y baja dotación intelectual, también se observa esta tendencia a no establecerse diferencias significativas entre las dos variables.

Pensamos que el desajuste entre lo que observamos en la práctica pedagógica diaria y los resultados de las mediciones que efectuamos estriba en las dificultades para evaluar correctamente las estrategias que utilizan los alumnos.

El procedimiento habitual para examinar las estrategias de aprendizaje de los estudiantes es el uso de escalas o cuestionarios. Hay que recordar, sin embargo, que este tipo de evaluación autopercibida, aún siendo de utilidad, presenta importantes inconvenientes. Como todos los instrumentos de autoevaluación están mediados por 
variables, que hay que analizar. Concretamente en el caso de las estrategias de aprendizaje es importante tener en cuenta el autoconcepto académico del alumnado; las expectativas que crea el ser evaluados por el profesor y el desconocimiento mismo de en qué consiste una determinada estrategia (Elices, Palazuelo, Del Caño, 2003).

Muchos estudiantes son estratégicos en su estudio, pero no conocen o no saben hacer un análisis introspectivo de sus procesos intelectuales, especialmente en los niveles de primaria. Además las estrategias de aprendizaje se utilizan simultáneamente y aunque hay una tendencia en cada persona a usar unas u otras, la propia materia de estudio determina su uso.

Otro importante aspecto a tener en cuenta estriba en el instrumento mismo con el que se evalúan las tácticas y estrategias de los alumnos. No es lo mismo obtener los datos de preguntas que se les formulan directamente o realizar estimaciones de la utilización de las mismas en contextos reales de estudio (Marugán, Del Caño, Román y Torres, 2007).

Creemos que nos hemos de emplear con tesón en diseñar instrumentos o procedimientos más adaptados a la realidad para efectuar dichas mediciones, en caso de no hacerlo así los diseños de entrenamientos en estrategias de aprendizaje serán difíciles de validar experimentalmente. Quizá por estas dificultades se opte por entrenar a profesores, esperando que estos sean los que trasmitan, con su modelado, una forma eficaz de incorporar estrategias de aprendizaje en el estudio, pero si no se mejora la evaluación del alumnado se desconocerá hasta qué punto la enseñanza ha sido eficaz.

La evaluación de las estrategias de aprendizaje de los alumnos, y en particular por lo que atañe a este trabajo, de los de alta capacidad, sigue siendo un tema pendiente de nuestras investigaciones. No descartamos el utilizar metodología cualitativa con entrevistas en profundidad a alumnos seleccionados de alta y no alta capacitación intelectual.

\section{BIBLIOGRAFÍA}

Beltrán, J. (1993). Procesos, estrategias y técnicas de aprendizaje. Madrid: Síntesis.

Beltrán, J. (1996). Estrategias de aprendizaje. En J. Beltrán \& C. Genovard (Eds.), Psicología de la Instrucción I: Variables y procesos básicos (pp. 383-428). Madrid: Síntesis

Beltrán, J., \& Genovard, C. (Eds.) (1999). Psicología de la Instrucción (Vol. III). Madrid: Síntesis.

Bernad, J. A. (1995). Estrategias de estudio en la universidad. Madrid: Síntesis

Cattell, R. B., \& Cattell, A. K. S. (1990). Test de Factor "g". Escala 2. Madrid: TEA.

De la Torre, S. (1991). Evaluación de la Creatividad. T.A.E.C. Un Instrumento de Apoyo a la Reforma. Madrid: Editorial Escuela Española.

Del Caño, M., Marugán, M., Román, J. M., Galán, M., \& Torres, H. (2005). Estrategias de aprendizaje y alumnos con altas capacidades. En I. Ruiz, F. Vicente, A. Ventura, J. A. Barrio, \& F. Fajardo (Eds.), Necesidades educativas específicas. ¿Hay respuestas? (pp. 65-72). Badajoz: Psicoex.

Doménech, M. (2004). El papel de la inteligencia y la metacognición en la resolución de problemas (Tesis doctoral). Recuperado de http://www.tesisenred.net/ bitstream/ handle/10803/8958/TesiintelimetacMontseDomenechp.pdf?sequence=1 
Elices, J. A., Palazuelo, M. M., \& Del Caño, M. (2003). Necesidades educativas del alumnado superdotado. Identificación y Evaluación. Junta de Castilla y León.

Gallego, J. L., \& González, J. (2008). Cómo planifican la composición escrita alumnos con sobredotación intelectual. Revista de Investigación Educativa, 26 (2), 463-484.

García, J. A., Prieto, M. D., \& Bermejo, M. R. (2000). Estrategias de aprendizaje en estudiantes más capaces. Faisca. Revista de Altas Capacidades, 8, 45-53.

García, R., Clemente, A., \& Pérez, E. (1992). Evolución y desarrollo de las estrategias de aprendizaje en Psicología de la Educación. Revista de Historia de la Psicología, 13, 1-17.

López, F. J. (1998). Programa de entrenamiento en estrategias de elaboración de metáforas para alumnos de secundaria (Tesis doctoral no publicada). Universidad Valladolid, Valladolid.

López, O., \& Navarro, J. (2010). Creatividad e inteligencia: un estudio en Educación Primaria. Revista de Investigación Educativa, 28 (2), 283-296.

Martín, L .J., Román, J. M., \& Carbonero, M. A. (2005). Eficacia de un Programa de entrenamiento en paráfrasis para la mejora del rendimiento. En J. A. Del Barrio, M. I. Fajardo, F. Vicente, A. Ventura, \& I. Ruiz (Eds.), Nuevos contextos psicológicos y sociales en educación: Buscando respuestas (pp. 409-424). Badajoz: Psicoex.

Marugán, M. (1996). Diseño y validación de un programa de entrenamiento en estrategias de relación para alumnos de enseñanza secundaria. Valladolid: Secretariado de Publicaciones e Intercambio Científico de la Universidad de Valladolid.

Marugán, M., \& Román, J. M. (1997). Aprendo si relaciono: Programa de entrenamiento en "estrategias de relación" para alumnos de Secundaria. Madrid: Visor.

Marugán, M., Del Caño, M., Román, M., \& Torres, H. (2007). Estudio comparativo entre la evaluación psicométrica y la identificación del profesor para variables psicoeducativas relevantes en primaria y secundaria. Revista de Psicología General y Aplicada, 60, 377-395.

Marugán, M. (2009). Importancia de las estrategias generales de aprendizaje en el rendimiento escolar. Quaderns Digitals: Revista de nuevas tecnologías y Sociedad, 55, 1-6

Monereo, C. (1993). Las estrategias de aprendizaje: Procesos, contenidos e interacción. Barcelona: Doménech.

Nisbet, J., \& Shucksmith, J. (1987). Estrategias de aprendizaje. Madrid: Santillana.

Preckel, F., Holling, H., \& Wiese, M. (2006). Relationship of intelligence and creativity in gifted and non - gifted students: An investigation of threshold theory. Personality and Individual Differences, 40, 159-170.

Prieto, M. D., \& Castejón, J. A. (1999). Recursos estratégicos en alumnos superdotados. En A. Sipán (Coord.), Respuestas educativas para alumnos superdotados y talentosos (pp. 211-243). Zaragoza: Mira Editores.

Raven, J. C. (1996). Test de matrices progresivas. Escala Color (CPM). Madrid: TEA.

Román, J. M., \& Gallego, S. (1994). ACRA: Escalas de Estrategias de Aprendizaje. Madrid: TEA. Sligh, A., Conners, F., \& Roskos, B. (2005). Relation of creativity to fluid and crystallized intelligence. The Journal of Creative Behaviour, 39 (2), 123-136.

Fecha de recepción: 16 de febrero de 2012.

Fecha de revisión: 16 de febrero de 2012.

Fecha de aceptación: 17 de mayo de 2012. 